\title{
OPEN PROBLEMS AND FUTURE PROSPECTS FOR HYPERNUCLEAR PHYSICS
}

\author{
Carl B. DOVER \\ Physics Department \\ Brookhaven National Laboratory \\ Upton, New York 11973, USA
}

\begin{abstract}
We appraise the current status of our knowledge of hypernuclear structure physics, and emphasize the unsolved problems. The prospects for significant advances in high resolution hypernuclear spectroscopy with CW electron beams at CEBAF or intense pion beams at the proposed PILAC facility at LAMPF are discussed. These facilities could greatly extend our understanding of strangeness $S=-1$ hypernuclear systems. For $S=-2$ systems, new events have been seen in a $\left(K^{-}, K^{+}\right)$hybrid counter-emulsion experiment at KEK in Japan. We give a theoretical interpretation of one of these events, as well as some further possibilities for the exploration of $\Lambda \Lambda$ hypernuclear spectroscopy via $\Xi^{-}$-atoms. We mention some possible enhancements of $\left(K^{-}, K^{+}\right)$or $\left(K^{-}, K^{0}\right)$ cross sections to discrete states, due to $\Xi N-\Lambda \Lambda$ configuration mixing in a shell model description of $S=-2$ hypernuclei. Finally, we explore the possibilities for producing multi-strange nuclei or droplets of strange quark matter ("strangelets") in relativistic heavy ion collisions.
\end{abstract}

Invited talk presented at the International Symposium on Hypernuclear and Strange Particle Physics

Shimoda, Japan - 9-12 December 1991

This manuscript has been authored under contract number DE-AC02-76C1100016 with the U.S. Department of Energy. Accordingly, the U.S. Government retains a non-exclusive, royalty-free license to publish or reproduce the published form of this contribution, or allow others to do so, for U.S. Government purposes.
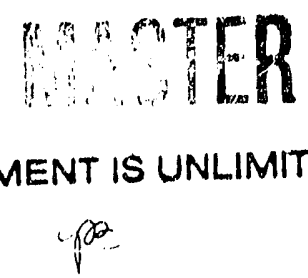
OPEN PROBLEMS AND FUTURE PROSPECTS FOR HYPERNUCLEAR PHYSICS

Carl B. Dover

Brookhaven National Laboratory, Physics Department, Upton, N.Y., 11973

\begin{abstract}
We appraise the current status of our knowledge of hypernuclear structure physics, and emphasize the unsolved problems. The prospects for significant advances in high resolution hypernuclear spectroscopy with CW electron beams at CEBAF or intense pion beams at the proposed PILAC facility at LAMPF are discussed. These facilities could greatly extend our understanding of strangeness $S=-1$ hypernuclear systems. For $S=-2$ systems, new events have been seen in a $\left(K^{-}, K^{+}\right)$hybrid counter-emulsion experiment at KEK in Japan. We give a theoretical interpretation of one of these events, as well as some further possibilities for the exploration of $\Lambda \Lambda$ hypernuclear spectroscopy via $\Xi^{-}$-atoms. We mention some possible enhancements of $\left(K^{-}, K^{+}\right)$or $\left(K^{-}, K^{0}\right)$ cross sections to discrete states, due to $\Xi N-\Lambda \Lambda$ configuration mixing in a shell model description of $S=-2$ hypernuclei. Finally, we explore the possibilities for producing multi-strange nuclei or droplets of strange quark matter ("strangelets") in relativistic heavy ion collisions.
\end{abstract}

\title{
1. INTRODUCTION
}

In this talk, we survey the field of hypernuclear physics. with an emphasis on open problems and the prospects for the further development of strange particle nuclear physics at future accelerator facilities. We start with a discussion of two-body hyperon-nucleon $(Y N)$ scattering processes, with emphasis on the spin dependence and $\mathrm{SU}(3)$ properties of these elementary interactions. We then appraise the status of our knowledge regarding the effective $\Lambda N$ interaction in the nuclear medium, focusing on recent analyses in the $p$-shell, the relation of effective and free space interactions, and the possible influence of spin-dependent three-body forces. The single particle structure of $\Lambda, \Sigma$ and possible $\Xi$ hypernuclei is discussed. For $\Lambda$-nucleus systems, enormous progress in structure studies could be made if high resolution facilities become available. We examine the prospects for CEBAF, PILAC and KAON in this regard.

The availability of higher momentum $(1.6-2 \mathrm{GeV} / \mathrm{c})$ beam lines at KEK and the Brookhaven AGS offers attractive possibilities for the study of strangeness $S=-2$ hypernuclei. We suggest an interpretation of a $\Lambda \Lambda$ hypernuclear event recently seen at $K E K$, and mention several other experiments which could shed further light on the nature of the $\Lambda \Lambda$ interaction and the existence of the $H$ dibaryon. An intriguing possibility is that $\Xi+A$ and $\Lambda \Lambda+(A-1)$

This manuscript has been authored under contract number DE-AC02-76CH00016 with the U.S. Department of Energy. 
states $(A=$ nuclear core) may enjoy a non-negligille mixing in some optimal cases, enhancing the $\left(K^{-}, K^{+}\right)$cross sections to particular $S=-2$ hypernuclear states.

Finally, we explore the possibilities for producing multi- $\Lambda$ hypernuclei or droplets of strange quark matter ("strangelets") in relativistic heavy ion collisions. Such experiments have been proposed at Brookhaven (E864) and at CERN.

\section{HYPERON-NUCLEON SCATTERING: SPIN OBSERVABLES AND SU(3)}

Compared to the abundant and precise data available for nucleon-nucleon $(N N)$ scattering, $Y N$ data ${ }^{1}$ are extremely limited. Essentially the world supply of data on $\Lambda N$ and $\Sigma N$ reactions is depicted in Fig. 1, taken from Ref. 2. The solid curves in Fig. 1 represent fits due to Nagels et al. ${ }^{3}$ (Model D), in which the one boson exchange (OBE) approximation is used to describe the $N N$ potential $V(r)$, supplemented by hard cores at short distances. The data can also be accommodated in a variety of other models, involving soft cores ${ }^{4}$ or one gluon exchange ${ }^{5}$ at small $r$ in coordinate space, or OBE models formulated in momentum space $^{6}$.

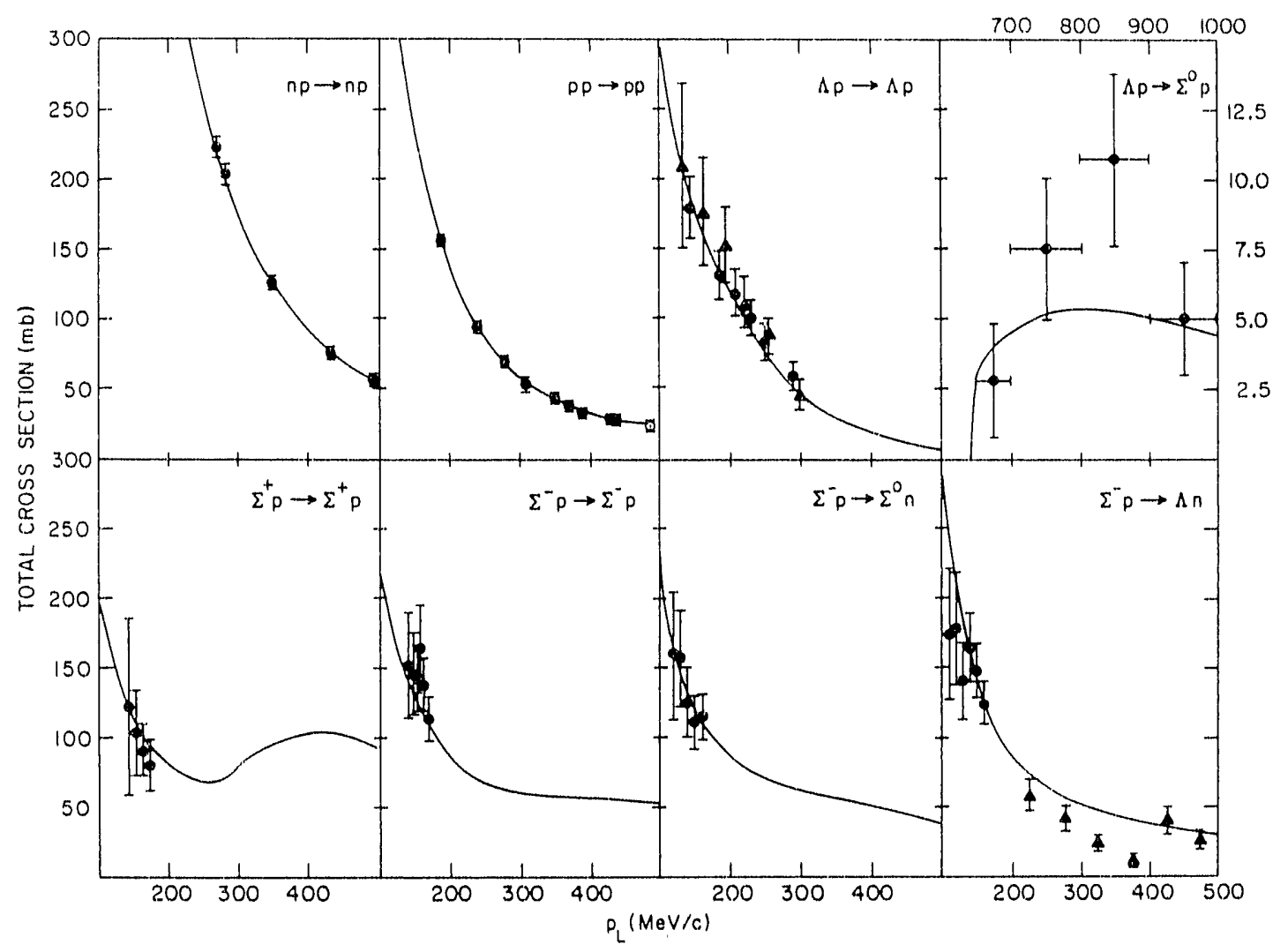

Figure 1: Baryon-baryon total cross sections as a function of lab momentum $p_{L}$, from Ref. 2. The data are from Ref. 1, and solid curves are potential model calculations of the Nijmegen group ${ }^{3}$. 
There are a number of physics questions associated with $Y N$ scattering which could be explored at future facilities such as the proposed PILAC (Pion Linear Accelerator) at; LAMPF or at the KAON factory in Vancouver. The main questions involve the spin dependence of the $Y N$ interaction and the degree to which $\mathrm{SU}(3)$ symmetry is respected in such interactions. Presently, there is very little information on spin observables for $Y N$ scattering: even the polarization $P(\theta)$ for elastic $\Lambda p$ scattering is essentially unknown. Existing models which fit equally well the spin-averaged $Y N$ data on total and differential cross sections predict quite different spin dependences for the $s$-wave $\Lambda p \rightarrow \Lambda p$ scattering lengths a. For instance, the Nijmegen hard core Models D, F yield ${ }^{3}$

$$
a\left({ }^{1} \mathrm{~S}_{0}\right) \approx a\left({ }^{3} \mathrm{~S}_{1}\right)
$$

whereas the more recent soft core OBE model ${ }^{4}$ predicts

$$
a\left({ }^{1} \mathrm{~S}_{0}\right) \approx-2.8 \mathrm{fm}, \quad a\left({ }^{3} \mathrm{~S}_{1}\right) \approx-1.4 \mathrm{fm}
$$

i.e., a sizable spin dependence. Data on $P(\theta)$ would reveal information on the spin-orbit coupling: note that for the $Y N$ system, unlike $N N$, one has both symmetric $\left[\left(\vec{\sigma}_{Y}+\vec{\sigma}_{N}\right) \cdot \vec{L}\right]$ and antisymmetric $\left[\left(\vec{\sigma}_{Y}-\vec{\sigma}_{N}\right) \cdot \vec{L}\right]$ spin-orbit terms. For some time, it has been known ${ }^{7}$ that the strength $V_{L S}^{\Lambda}$ of the effective one-body $\Lambda$-nucleus spin-orbit potential is very weak. Polarization data would be invaluable in clarifying the relation between the two-body $\Lambda N$ spin-orbit interaction and the small value of $V_{L S}^{\Lambda}$. Other aspects of the spin dependence are equally interesting, for instance the $\vec{\sigma}_{\Lambda} \cdot \vec{\sigma}_{N}$ and tensor terms $\left(S_{12}\right)$. The $\left(K^{-}, \pi^{-} \gamma\right)$ data on hypernuclei ${ }^{8}$ have led to some constraints on these terms in the $\Lambda N$ effective interaction $G$. However, attempts to relate $G$ to the free space potential $V_{N \Lambda}$ have not been very successful in correlating the information on spin splittings of hypernuclear levels.

The rôle of $\mathrm{SU}(3)$ symmetry in baryon-baryon (BB) scattering would be greatly illuminated if spin-separated cross section $\sigma_{S}$ were available. For $s$-waves, for instance, we have ${ }^{2}$ the $\mathrm{SU}(3)$ relations

$$
\begin{aligned}
\sigma_{0}\left(\Sigma^{+} p \rightarrow \Sigma^{+} p\right) & =\sigma_{0}(n p \rightarrow n p) \\
\sigma_{0}\left(\Sigma^{-} p \rightarrow \Lambda n\right) & =\frac{1}{3} \sigma_{0}\left(\Sigma^{-} p \rightarrow \Sigma^{0} n\right) \\
\sigma_{0}(\Lambda p \rightarrow \Lambda p) & =\frac{1}{6}\left[5 \sigma_{0}\left(\Sigma^{+} p \rightarrow \Sigma^{+} p\right)+\sigma_{0}\left(\Sigma^{-} p \rightarrow \Sigma^{-} p\right)-\frac{5}{3} \sigma_{0}\left(\Sigma^{-} p \rightarrow \Sigma^{0} n\right)\right] \\
\sigma_{1}(\Lambda p \rightarrow \Lambda p) & =\frac{1}{2}\left[3 \sigma_{1}\left(\Sigma^{-} p \rightarrow \Sigma^{-} p\right)+3 \sigma_{1}\left(\Sigma^{-} p \rightarrow \Sigma^{0} n\right)-\sigma_{1}\left(\Sigma^{+} p \rightarrow \Sigma^{+} p\right)\right] \\
\sigma_{0}\left(\Xi^{-} p \rightarrow \Xi^{0} n\right) & : \sigma_{0}\left(\Xi^{-} p \rightarrow \Sigma^{0} \Lambda\right): \sigma_{0}\left(\Xi^{-} p \rightarrow \Lambda \Lambda\right)=1: 3: \frac{9}{2}
\end{aligned}
$$

In OBE models, one usually assumes SU(3) symmetry for coupling constants, and breaks the symmetry through the use of observed meson and baryon masses in constructing $B B$ potentials. This procedure yiclds good fits to the sparse data on spin-averaged cross sections, but one is not able to test the relations of Eq. (3). Theoretical studies of the mechanisms of 
$\mathrm{SU}(3)$ symmetry breaking suggest that it should be largely confined to diagonal transitions, i.e., those involving $\Lambda p \rightarrow \Lambda p$.

\section{THE $\Lambda N$ EFFECTIVE INTERACTION}

In the hypernuclear shell model, we diagonalize the Hamiltonian

$$
H=H_{N}+H_{\Lambda}+V_{N \Lambda}+V_{N N \Lambda}
$$

In the weak coupling basis, the nuclear the $\Lambda$ single particle terms $H_{N}$ and $H_{\Lambda}$ are already diagonal and the energies can be obtained from experiment. Generally, phenomenological two and three-body effective residual interactions $V_{N \Lambda}$ and $V_{N N \Lambda}$ have been used to explore the consequences of configuration mixing and to fit binding energies. The elimination of the $\Sigma$ channel (arising from $\Lambda N \rightarrow \Sigma N$ coupling) gives contributions to both $V_{N \Lambda}$ and $V_{N N \Lambda}$. The systematics of binding energies indicate that a repulsive $V_{N N \Lambda}$ is needed. This threebody term may also have a spin dependence; in fact, Bodmer and Usmani ${ }^{10}$ attribute about $1 / 3$ of the $1.1 \mathrm{MeV}$ separation of the $0^{+}-1^{+}$ground state doublet in ${ }_{\Lambda}^{4} \mathrm{H}-{ }_{\Lambda}^{4} \mathrm{He}$ to the spin dependence of $V_{N N \Lambda}$.

Consider $p$-shell hypernuclei with a $\Lambda$ in the $s$ orbit. The $p_{N} s_{\Lambda}$ effective interaction can be expressed in terms of five radial integrals $\bar{V}, \Delta, S_{\Lambda}, S_{N}$ and $T$, corresponding to the five terms in

$$
V_{N \Lambda}=V_{0}+V_{\sigma} \vec{s}_{N} \cdot \vec{s}_{\Lambda}+V_{\Lambda} \vec{\ell}_{N \Lambda} \cdot \vec{s}_{\Lambda}+V_{N} \vec{\ell}_{N \Lambda} \cdot \vec{s}_{N}+V_{T} S_{12}
$$

Ground state doublet energy splittings are given by the linear combinations

$$
\begin{aligned}
\delta & =\frac{2}{3} \Delta+\frac{4}{3} S_{\Lambda}-\frac{8}{5} T \quad\left(p_{3 / 2}^{N} s_{1 / 2}^{\Lambda}\right) \\
\delta^{\prime} & =-\frac{1}{3} \Delta+\frac{4}{3} S_{\Lambda}+8 T\left(p_{1 / 2}^{N} s_{1 / 2}^{\Lambda}\right)
\end{aligned}
$$

Based on the OBE Nijmegen Model D, Millener et al. ${ }^{11}$ developed a standard interaction with $\Delta=0.50, S_{\Lambda}=-0.04, S_{N}=-0.08, T=0.04$ (in $\mathrm{MeV}$ ), corresponding to $\delta=220$ $\mathrm{keV}, \delta^{\prime}=100 \mathrm{keV}$. Thus doublet splittings, and hypernuclear spin effects in general, are expected to be small. One prediction of Ref. 11 is that the $2^{--}-1^{-}$doublet splitting in ${ }_{\Lambda}^{10} \mathrm{~B}$ should be about $\Delta E=170 \mathrm{keV}$; Chrien et al. ${ }^{8}$ found $\Delta E<100 \mathrm{keV}$, so the spin splittings in hypernuclei appear to be even smaller than foreseen. Fetisov et al. ${ }^{12}$ recently undertook a re-analysis of hypernuclear spin splittings, including the recent data, and proposed a parameter set $\Delta=0.3, S_{\Lambda}=-0.02, S_{N}=-0.35\left({ }_{\Lambda}^{7} \mathrm{Li}\right),-0.1(A>7), T=0.02 \mathrm{MeV}$, with a weaker spin dependence than Ref. 11.

A number of open questions remain: Is there a universal set of $\Lambda N$ matrix elements $\left(\bar{V}, \Delta, S_{\Lambda}, S_{N}, T\right)$ in the $p$-shell which enable us to describe the observed spin splittings? Does the spin depondence of the $V_{N N \Lambda}$ play a significant role? What is the relation of $V_{N \Lambda}$ to the free space $\Lambda N$ interaction? We need further experimental information on spin splittings in order to overconstrain the two-body $V_{N \Lambda}$ : only then can we decisively test 
the adequacy of the simple description with universal matrix elements. High resolution $\left(K^{-}, \pi^{-}\right),\left(\pi^{+}, K^{+}\right)$or $\left(\gamma, K^{+}\right)$studies at KAON, PILAC or CEBAF would qualitatively advance our knowledge of the $\Lambda$ interaction in nuclei. Studies of hypernuclear $\gamma$ rays, after formation of various excited states in one of the above reactions, are another key component. We now offer some comments on future possibilities for such efforts.

\section{PROSPECTS FOR HIGH RESOLUTION HYPERNUCLEAR STRUCTURE STUDIES}

To make qualitative progress in hypernuclear structure physics, we need to resolve the fine structure splittings, and this requires high resolution facilties. Hypernuclear spin splittings are small ( $\leq 500 \mathrm{keV}$ ), so an energy resolution of order $\Delta E \approx 200 \mathrm{keV}$ is highly desirable. With the advent of the continuous beam (CW) electron accelerator CEBAF, it becomes feasible to perform hypernuclear experiments with real $[(\gamma, K)]$ or virtual $\left[\left(e, e^{\prime} K\right)\right]$ photons. Another extremely promising alternative would be afforded by very intense pion beams in the $1 \mathrm{GeV} / \mathrm{c}$ region, for instance from PILAC, the pion linear accelerator ${ }^{13}$ being discussed for construction at LAMPF. We discuss these possibilities in turn.

The $(\bar{K}, \pi)$ amd $(\pi, K)$ reactions on spin zero targets excite only non-spin-flip natural parity $\left(0^{+}, 1^{-}, 2^{+}\right.$, etc. $)$states at $0^{\circ}$. The $(\gamma, K)$ process, on the other hand, preferentially excites unnatural parity states, and is thus complementary to the hadronic reactions. The leading momentum-independent part of the $\gamma N \rightarrow K \Lambda$ transition operator is proportional to $\vec{\sigma} \cdot \hat{\epsilon}$, where $\vec{\sigma}$ and $\hat{\epsilon}$ are the nucleon spin and photon polarization, respectively. This term dominates for transitions to particle-hole configurations $\left(j_{N}^{-1} j_{\Lambda}\right) J$, for nodeless $j=\ell+1 / 2$ orbits with maximum $J=\ell_{N}+\ell_{\Lambda}+1$. For weaker transitions, configuration mixing as well as the momentum dependent terms in the $(\gamma, K)$ operator are likely to be significant.

Results of particle-hole calculations ${ }^{14}$ for the reaction ${ }^{12} \mathrm{C}\left(\gamma, K^{+}\right){ }_{\Lambda}^{12} \mathrm{~B}^{*}$ are shown in Fig. 2. Contributions from the $\left(s_{1 / 2}^{\Lambda} p_{3 / 2}^{-1}\right),\left(p_{1 / 2,3 / 2}^{\Lambda} p_{3 / 2}^{-1}\right),\left(s_{1 / 2}^{\Lambda} s_{1 / 2}^{-1}\right)$ and $\left(p_{1 / 2,3 / 2}^{\Lambda} s_{1 / 2}^{-1}\right)$ configurations are included. The largest $\left(\gamma, K^{+}\right)$cross section corresponds to the highest spin $\left(p_{3 / 2}^{\Lambda} p_{3 / 2}^{-1}\right)_{3}+$ state, which is excited with negligible strength in the $\left(\pi^{+}, K^{+}\right)$or $\left(K^{-}, \pi^{-}\right)$reaction on ${ }^{12} \mathrm{C}$. The strengths of the two components of the ground state doublet $\left(s_{1 / 2}^{\Lambda} p_{3 / 2}^{-1}\right)_{1-, 2-}$ are seen to be comparable, and hence one may be able to measure their splitting directly in a high resolution $(\gamma, K)$ experiment. Except for the measured $1.1 \mathrm{MeV}$ splitting ${ }^{15}$ of the $\left(s_{1 / 2}^{\Lambda} s_{1 / 2}^{-1}\right)_{0+, 1}+$ configurations in ${ }_{\Lambda}^{4} \mathrm{He}$ and ${ }_{\Lambda}^{4} \mathrm{H}$, no other doublet splittings are known.

Another favorable case for CEBAF is the ${ }^{9} \mathrm{Be}\left(\gamma, K^{+}\right){ }_{\Lambda}^{9} \mathrm{Li}$ reaction. The doublet splittings in ${ }_{\Lambda}^{9} \mathrm{Li}$ for the coupling of $s_{1 / 2}^{\Lambda}$ to the $2^{+}$(g.s.) and $1^{+}$core states of ${ }^{8} \mathrm{Li}$ are predicted ${ }^{16}$ to be

$$
\Delta E\left(s_{1 / 2}^{\Lambda}\right)= \begin{cases}1.21 \Delta+1.21 S_{\Lambda}+1.00 T & \left(s_{1 / 2}^{\Lambda} \otimes 2^{+}\right) \\ 0.69 \Delta+0.73 S_{\Lambda}-3.3 T & \left(s_{1 / 2}^{\Lambda} \otimes 1^{+}\right)\end{cases}
$$

The largest contribution to $\Delta E$ arises from $\Delta$, i.e., the $\vec{\sigma}_{N} \cdot \vec{\sigma}_{\Lambda}$ interaction. The splitting $\Delta E \approx 0.5 \mathrm{MeV}$ of the ground state ${ }_{\Lambda}^{9} \mathrm{Li}$ doublet should be measurable at CEBAF. Many 


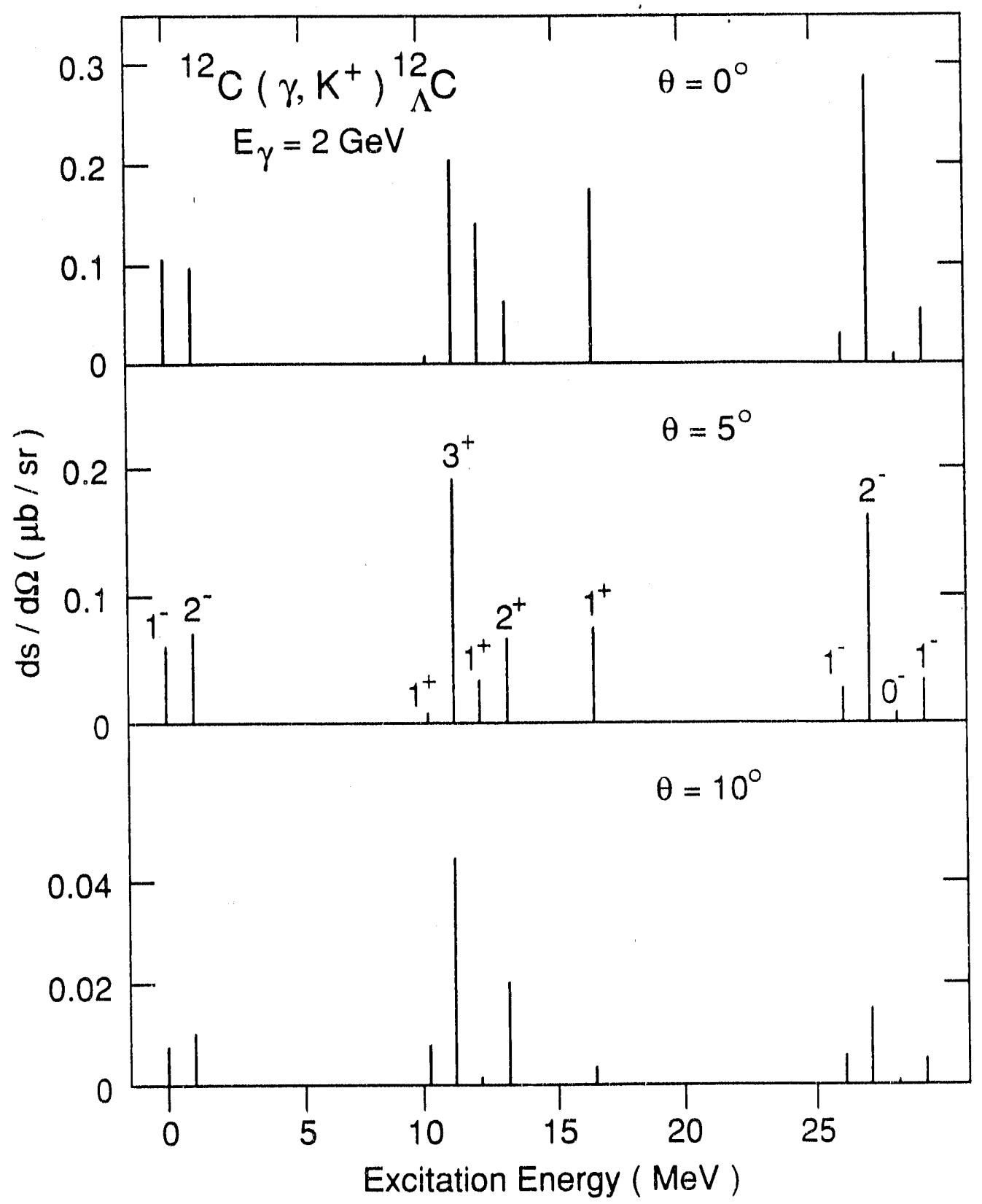

Figure 2: Predicted differential cross sections ${ }^{14}$ for the reaction ${ }^{12} \mathrm{C}\left(\gamma, K^{+}\right)$ ${ }_{\Lambda}^{12} \mathrm{~B}^{*}$ at $2 \mathrm{GeV}$ and various kaon angles $\theta_{K}$. Note the dominance of spin-flip transitions to unnatural parity $\left(2^{-}, 3^{+}\right)$particle-hole states.

other $s_{\Lambda}$ doublet splittings are probably too small to be measured directly, even with a resolution $\Delta=200 \mathrm{keV}$. In most cases, however, unresolved peaks due to doublets based on different nuclear core states should be well separated: there is information on $V_{N \Lambda}$ to be gained from the energy separation of such peaks and the magnitude of the cross sections as a function of energy and momentum transfer.

The proposed pion linear accelerator (PILAC) at LAMPF offers the exciting prospect of high resolution $\left(\pi^{+}, K^{+}\right)$reaction studies ${ }^{13}$, a necessary complement to the $\left(\gamma, K^{-}\right)$experiments at CEBAF. PILAC would employ high-gradient superconducting cavities to accelerate 
pions from LAMPF to the vicinity of $1 \mathrm{GeV} / \mathrm{c}$, providing $10^{9} \pi^{+} / \mathrm{sec}$. on target and $200 \mathrm{keV}$ resolution. This facility would generate an unprecedented intensity of pions at a momentum near the peak of the $\pi^{+} n \rightarrow K^{+} \Lambda$ cross section. The physics motivation for PILAC is rather broad, and was developed in detail in a recent Workshop ${ }^{13}$. In addition to the $\left(\pi^{+}, K^{+}\right)$ studies emphasized here, we also mention the possibilities for detailed investigations of the structure and decays of $N^{*}$ and $\Delta^{*}$ resonances, and the study of the interactions and decays of $\eta$ mesons (a tagged $\eta$ beam can be produced via the $\pi^{-} p \rightarrow \eta n$ process).

At KAON, the availability of high intensity kaon and pion beams would also permit high resolution $\left(K^{-}, \pi^{-}\right)$and $\left(\pi^{+}, K^{+}\right)$experiments, as well as precision measurements of hypernuclear $\gamma$ rays. However, it is difficult to gauge the relative priority of hypernuclear physics at KAON, i.e., in the context of a facility which is largely focused on particle physics questions. We would argue that the case for hypernuclear physics research at KAON is a compelling one, not to be relegated to the rôle of a satellite activity.

\section{HYPERON SINGLE PARTICLE STATES IN NUCLEI}

Even with coarse resolution $\left(\pi^{+}, K^{+}\right)$and $\left(K^{-}, \pi^{-}\right)$experiments, considerable information has been obtained on $\Lambda$ single particle structure ${ }^{17}$. Here we sum over the fine structure of levels split by spin dependent interactions. The $s_{\Lambda}, p_{\Lambda}, d_{\Lambda}$, and $f_{\Lambda}$ binding energies display a smooth dependence on mass number $A$. The absence of shell effects, in contrast to the case of nucleon single particle states, is consistent with the notion that the $\Lambda$ behaves as a distinguishable particle in the nucleus.

The spacings of the $\Lambda$ single-particle levels for a given $A$ constrain both the radius and the depth of the $\Lambda$-nucleus potential well. The data have been analyzed with a phenomenological $\Lambda$ potential ${ }^{18}$ based on the spherical Skyrme-Hartree-Fock approach. A similar approach is due to Yamamoto et al. ${ }^{19}$. The essential features of the potential are an attractive component linear in the nuclear density $\rho(r)$, and a repulsive component proportional to a higher power of the density. The first order term has a depth of $60 \mathrm{MeV}$, consistent with estimates based on the free space $\Lambda N$ interaction. Here, the non-locality of the $\Lambda$ potential is parametrized in terms of an effective mass $n_{\Lambda}^{*}(r)$. The equivalent energy-dependent local potential $V_{\Lambda}(r, E)$ is of the form

$$
\begin{aligned}
V_{\Lambda}(r, E) & =\frac{m_{\Lambda}^{*}(r)}{m_{\Lambda}} U(r)+\left(1-\frac{m_{\Lambda}^{*}(r)}{m_{\Lambda}}\right) E \\
U(r) & =t_{0} \rho(r)+\frac{3}{8} t_{3} \rho^{2}(r)+\frac{1}{4}\left(t_{1}+t_{2}\right) T(r) \\
T(r) & =\frac{3}{5}\left(\frac{3 \pi^{2}}{2}\right)^{2 / 3} \rho^{5 / 3}(r) \\
\frac{\hbar^{2}}{2 m_{\Lambda}^{*}(r)} & =\frac{\hbar^{2}}{2 m_{\Lambda}}+\frac{1}{4}\left(t_{1}+t_{2}\right) \rho(r)
\end{aligned}
$$


The $t_{3} \rho^{2}(r)$ term can be used to adjust the radius of the potential, while the energy dependent term serves to spread out the single particle levels. This enables one to simultaneously fit the spectra of light $\left({ }_{\Lambda}^{16} \mathrm{O}\right)$ and heavy $\left({ }_{\Lambda}^{89} \mathrm{Y}\right)$ systems, which is not possible with a potential linear in $\rho(r)$ (assuming the parameters of $\rho(r)$ are fit to electron scattering data). The resulting $\Lambda$ well depth is around $28 \mathrm{MeV}$ and the radius is about $0.5 \mathrm{fm}$ larger than that of the underlying density, due to the non-linear terms in $\rho(r)$. An effective mass $m_{\Lambda}^{*}(0) / m_{\Lambda} \approx 0.8$ in the nuclear interior is needed to fit level spacings over the full mass range.

A single set of parameters in the Skyrme-Hartree-Fock picture of Eq. (8) suffices to simultaneously describe deeply and loosely bound $\Lambda$ orbitals. This is not the case for nucleon states. The $\Lambda$ appears to behave as a distinguishable particle in the nucleus, providing a superb example of single particle structure. Ünlike deeply bound nucleon-hole states, which are very broad, deeply bound $\Lambda$ states remain well defined. The possibility that the strange quark in the $\Lambda$ is partially deconfined in the nucleus is intriguing, but the signature of this effect in the $\Lambda$ binding energies is subtle, and easily masked by the conventional dy namics of density-dependent interactions. However, if $\Lambda$ level spacings are determined with greater precision, which could be accomplished at CEBAF or PILAC, one would more stringently test the standard mean field picture.

In contrast to the $\Lambda$, single particle states of other hyperons $(\Sigma, \Xi)$ undergo strong conversion in the nucleus $(\Sigma N \rightarrow \Lambda N, \Xi N \rightarrow \Lambda \Lambda)$, so they will be rather broar in most cases. The controversial evidence for narrow $\Sigma$ states $(\Gamma<5-10 \mathrm{MeV})$ is reviewed in Ref. 20 . In particular, several mechanisms have been discussed which could produce narrow states, including binding and Pauli effects, spin selectivity, and SU(3) selection rules. The recent data on ${ }_{\Sigma}^{4} \mathrm{He}$, which indicates a structure near the $\Sigma$ threshold, has provoked considerable discussion, focusing on the question of whether this bump should be interpreted as a cusp effect or a true bound state.

A number of questions remain open regarding $\Sigma$ states:

1. Do narrow structures near or below the $\Sigma$ threshold exist only for small $A$ ?

2. Are the narrow bumps seen in the $\Sigma$ continuum in early CERN experiments indeed real?

3. How does one reconcile the rather substantial well depth $D_{\Sigma} \approx 15-20 \mathrm{Me}$, deduced from $\Sigma^{-}$-atom level shifts ${ }^{20}$, with the very small values of $D_{\Sigma}$ obtained from DWBA analysis of $\left(K^{-}, \pi^{-}\right)$data?

If quasi--stable $\Sigma$ states are eventually shown to exist in a number of cases, one could address a number of fascinating questions involving their structure, for instance:

1. What is the isospin purity of $\Sigma$ states?

2. What is the spin-orbit potential for a $\Sigma$ ?

In OBE models, we anticipate a strong isospin dependence of the $\Sigma N$ interaction, and consequently a substantial Lane potential $\left(\vec{t}_{\Sigma} \cdot \vec{T}\right)$ which would tend to produce states of approximately good isospin. The spin-orbit strength $V_{L, S}^{\mathbb{D}}$ has been discussed both in quarkgluon and meson exchange models, which provide somewhat different predictions ${ }^{20}$. 
A similar set of questions can be posed for $\Xi$ hypernuclei. There is very little known about such objects, but there are some candidates in the literature. $\Xi$ single particle states would be accessible in the $\left(K^{-}, K^{+}\right)$or $\left(K^{-}, K^{0}\right)$ reactions on nuclear targets. A survey experiment of this type would be worthwhile, using the $K^{-}$beams available at KEK or the Brookhaven AGS.

\section{PRODUCTION AND STRUCTURE OF $S=-2$ HYPERNUCLEI}

We anticipate the existence of an extensive spectroscopy of doubly strange hypernuclei, to date largely unexplored. These include the $\Xi$ quasi-particle excitations mentioned in Section 5 , configurations containing two $\Lambda$ 's in bound states around a nuclear core, and possibly some states involving a non-negligible mixing of $\Xi$ and $\Lambda \Lambda$ configurations due to the $\Xi N \rightarrow \Lambda \Lambda$ transition potential.

The study of $S=-2$ hypernuclei is of considerable intrinsic interest, since one can gain information on the $\Lambda \Lambda$ effective interaction, and hence explore the $\mathrm{SU}(3)$ character of the strong forces of QCD. The study of such systems is also interesting from another point of view, since it can be argued that the observation of weak decays of $\Lambda \Lambda$ hypernuclei rules out the existence of a deeply bound $H$ dibaryon $^{21}$, a hypothetical six quark (ssuudd) state with $J^{\pi}=0^{+}$and $I=0$.

Evidence for the existence of the hypernuclei ${ }_{\Lambda \Lambda}^{6} \mathrm{He}$ and ${ }_{\Lambda \Lambda}^{10} \mathrm{Be}$ was reported in early emulsion experiments ${ }^{22}$. At $\mathrm{KEK}$, in recent experiments by Aoki et al. ${ }^{23}$ with a $1.66 \mathrm{GeV} / \mathrm{c}$ $K^{-}$beam, an event was seen which is consistent with the formation and decay of ${ }_{\Lambda \Lambda}^{10} \mathrm{Be}$ or ${ }_{\Lambda \Lambda}^{13} \mathrm{~B}$. In Ref. 24 , the ${ }_{\Lambda \Lambda}^{13} \mathrm{~B}$ hypothesis was judged to be more likely on physical grounds. Following the detection of the $K^{+}$from the $K^{-} p \rightarrow K^{+} \Xi^{-}$reaction, wilich was used to tag the formation of the $\Xi^{-}$, the reaction proceeds as follows:

$$
\begin{aligned}
\Xi^{-}+{ }^{14} \mathrm{~N} & \rightarrow n+{ }_{\Lambda \Lambda}^{14} \mathrm{C}^{*} \rightarrow n+p+{ }_{\Lambda \Lambda}^{13} \mathrm{~B} \\
{ }_{\Lambda \Lambda}^{13} \mathrm{~B} & \rightarrow \pi^{-}+{ }_{\Lambda}^{13} \mathrm{C} \\
{ }_{\Lambda}^{13} \mathrm{C} & \rightarrow 2 n+{ }^{3} \mathrm{He}+{ }^{4} \mathrm{He}+{ }^{4} \mathrm{He}
\end{aligned}
$$

Eq. (9a) corresponds to a sequence of two-body decays, proceeding through a relatively long-lived excited state

$$
{ }_{\Lambda \Lambda}^{14} \mathrm{C}^{*} \approx(1 s)_{\Lambda}(1 p)_{\Lambda} \otimes{ }^{12} \mathrm{C}^{*}(T=1)
$$

at about $24 \mathrm{MeV}$ of excitation. Here ${ }^{12} \mathrm{C}^{*}$ is an isovector core state (the $1^{+}$at about 15.1 $\mathrm{MeV}$ or the $2^{+}$at $16.1 \mathrm{MeV}$ ). The attractive $\Lambda \Lambda$ interaction energy $\Delta B_{\Lambda \Lambda}=4.8 \pm 0.7 \mathrm{MeV}$ (an $\left(s_{\Lambda}\right)^{2}$ matrix element) extracted from the data is consistent with eivilier results ${ }^{22}$ from

$\Lambda{ }_{\Lambda}^{6} \mathrm{He}$ and ${ }_{\Lambda \Lambda}^{10} \mathrm{Be}$. For comparison, we note that the corresponding ${ }^{1} \mathrm{~S}_{0}\left(s_{N}\right)^{2}$ matrix element is 5-i3 $\mathrm{MeV}$, while the $\left(s_{\Lambda} s_{N}\right) \Lambda N$ matrix element (from the ${ }_{\Lambda}^{5} \mathrm{He}$ binding energy) is $2-3$ $\mathrm{MeV}$. Thus the $\Lambda \Lambda\left({ }^{1} \mathrm{~S}_{0}\right)$ interaction seems to be more attractiv than that for $\Lambda N\left({ }^{1} \mathrm{~S}_{0}\right)$, and comparable to $N N\left({ }^{1} \mathrm{~S}_{0}\right)$. 
The relatively large value of $\Delta B_{\Lambda \Lambda}$ poses an interesting theoretical problem. The ${ }^{1} \mathrm{~S}_{0}$, $S=-2$ dibaryon interaction presents a rather special situation in the quark model, because of the possibility of a bound state, the $H$. In the $\mathrm{SU}(3)$ limit, the $H$ corresponds to the unitary singlet combination of $\Lambda \Lambda, \Sigma \Sigma$, and $\Xi N$ chennels at short distances. In the meson exchange picture, the ${ }^{1} \mathrm{~S}_{0}(\Lambda \Lambda \rightarrow \Lambda \Lambda)$ interaction is attractive ${ }^{24}$, and we do not expect strong short distance repulsion due to quark-gluon exchange, due to the strong channel coupling. There is also the possibility of a loosely bound $\Lambda \Lambda$ state near threshold, arising from such attractive meson exchange forces.

Other $\Xi^{-}$capture reactions at rest also offer promising prospects for making double hypernuclei. For instance, Zhu et $a .^{25}$ have predicted a branching ratio of about $3 \%$ for the process

$$
\Xi^{-}+{ }^{6} \mathrm{Li} \rightarrow{ }_{\Lambda}^{6} \mathrm{He}+n
$$

Targets of ${ }^{10} \mathrm{~B}$ or ${ }^{14} \mathrm{~N}$ may also be feasible. In such two-body reactions, one need only detect the mono-energetic neutron. The expected form of the neutron spectrum is shown in Fig. 3.

For heavier $p$-shell targets, the $\left(1 s_{\Lambda}\right)^{2}$ ground state yields are suppressed, and $\left(1 s_{\Lambda} 1 p_{\Lambda}\right)$ states are more strongly populated; Eq. (9a) is an example of this. Another experimental approach would consist of looking for $\gamma$ rays emitted from $\Lambda \Lambda$ excited states; some examples are provided in Ref. 25.

For heavier target nuclei beyond the $p$-shell we expect particle-unstable $\Lambda \Lambda$ configurations to be directly formed in $\Xi^{-}$capture at rest. These will decay by emitting nuclear fragments, an Auger-like cascade process by which the hyperons may deexcite to particlestable configurations in the daughter hypernuclei.

In the method just discussed, the $\Xi^{-}$was slowed down electromagnetically, and then captured in an atomic orbit around a nucleus, finally producing the $S=-2$ hypernucleus following a further $\Xi N \rightarrow \Lambda \Lambda$ reaction. An alternative is to form a $\Lambda \Lambda$ hypernucleus directly via a second order process on a nuclear target, as depicted in Fig. 4. We further note that in the $\left(K^{-}, \pi^{+}\right)$or $\left(\pi^{--}, K^{+}\right)$double charge exchange processes, such second order contributions would lead to a new class of $\Lambda$ hypernuclear states, not accessible in the usual $\left(\mathrm{K}^{-}, \pi^{-}\right)$or $\left(\pi^{+}, K^{+}\right)$reactions. The $\left(\pi^{-}, K^{+}\right)$process could be exploited at PILAC, for instance.

For a few simple cases, the diagram of Fig. (4a) was evaluated in Ref. 26. Since the process is second order, the $\left(K^{-}, K^{+}\right)$cross sections to discrete $\Lambda \Lambda$ hypernuclear states are rather small, of the order of a few $\mathrm{nb} / \mathrm{sr}$. A typical excitation energy spectrum is displayed in Fig. 5. Due to the substantial momentum transfer even at $\theta=0^{\circ}$, excitation of states of the highest available spin ( $4^{-1}$ in Fig. 5) is preferred.

The excitation of $\Xi$-hypernuclear states occurs via a single step process, with cross sections ${ }^{27}$ of order 100-1000 times larger than those shown in Fig. 5, typically of order 0.1$3 \mu \mathrm{b} / \mathrm{sr}$. However, these states are generally expected to be broad (perhaps with widths $\Gamma \sim 5-10 \mathrm{MeV}$ ), Decause of the strong conversion process $\Xi N \rightarrow \Lambda \Lambda$. An intriguing possibility exists that the configuration mixing of $\Xi+A$ and $\Lambda \Lambda+(A-1)$ states might lead to enhanced cross sections to low-lying particle-stable $S=-2$ eigenstates. Even though 


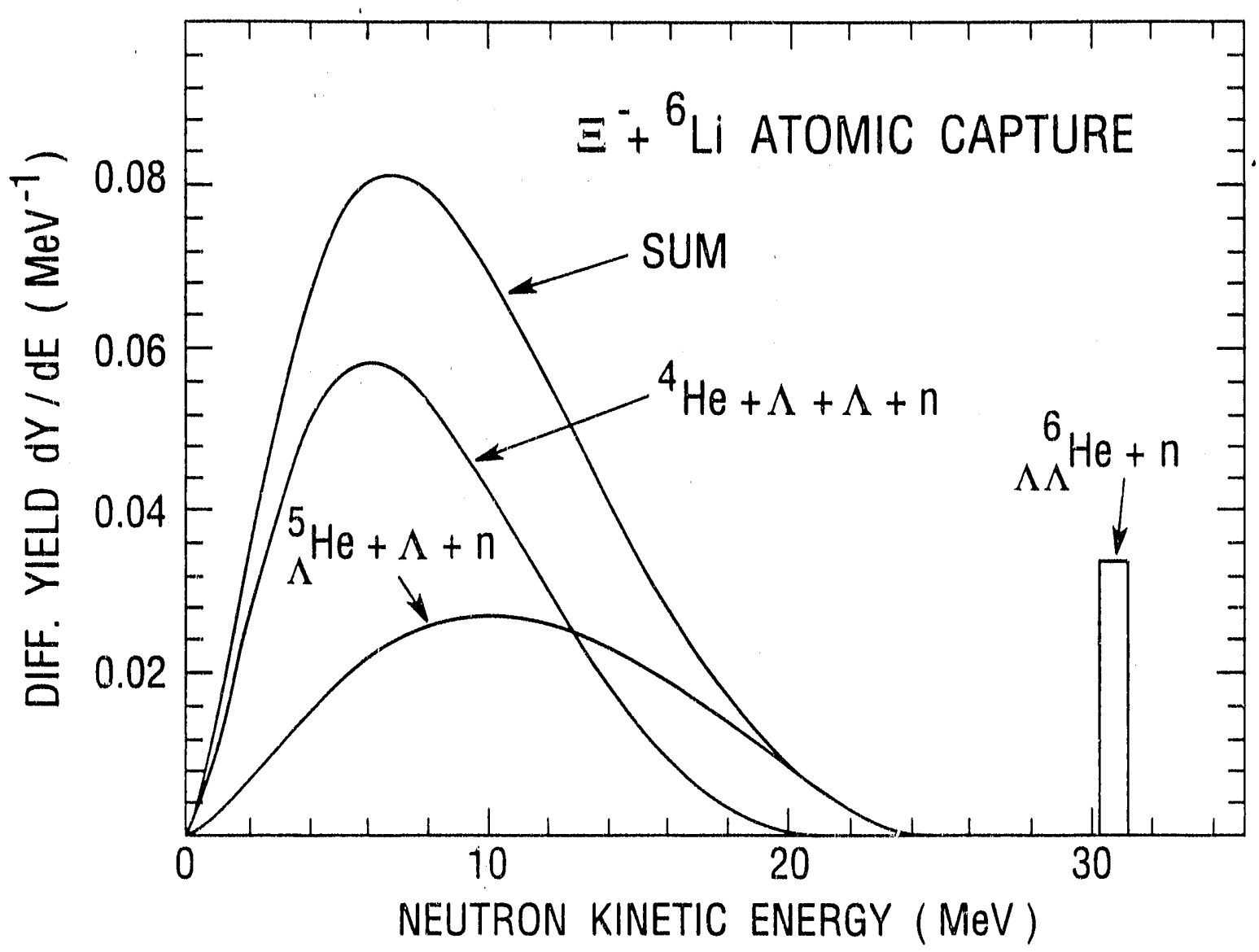

Figure 3: Calculated differential yield $d Y / d E$ as a function of the outgoing neutron kinetic energy for $\Xi^{-}+{ }^{6} \mathrm{Li}$ capture at rest. The contributions of the various reaction branches, as well as their sum, are indicated, assuming $20 \% p$ capture and $80 \% d$ capture. Assuming $1 \mathrm{MeV}$ energy resolution for the neutron, the contribution of the $n+{ }_{\Lambda}{ }^{6} \mathrm{He}$ channel is shown as a rectangle centered at $30.7 \mathrm{MeV}$; the integrated yield for this branch is about $3.4 \%$ per stopped $\Xi^{-}$, as estimated by Zhu et al. ${ }^{25}$.

these low-lying states remain dominantly of $\Lambda \Lambda+(A-1)$ character, the small $\Xi$ admixture brings the large first order cross section into the picture. Calculations are underway ${ }^{28}$ to assess the effects of $\Xi-\Lambda \Lambda$ configuration mixing in the $p$-shell, with the idea of isolating some optimal cases for experimental study. For $A=5$, some calculations along these lines were reported by Akaishi and Myint ${ }^{29}$. As an example, consider the ${ }^{15} \mathrm{~N}\left(K^{-}, K^{+}\right){ }_{\Lambda \Lambda}^{15} \mathrm{~B}^{*}$ reaction. Some of the energy levels and particle emission thresholds are depicted in Fig. 6 . The free space mass difference $m \Xi+m_{N}-2 m_{\Lambda} \approx 28.5 \mathrm{MeV}$ is reduced to $\Delta E \approx 15 \mathrm{MeV}$ in this case, assuming the $s \Xi-$ is bound by about $12.5 \mathrm{MeV}$ in its single particle potential ${ }^{27}$. In general, the neutron emission threshold will lie lowest in systems formed via the $\left(\mathrm{K}^{-}, \mathrm{K}^{+}\right)$ reaction, since two protons have been removed from the target.

To calculate the mixing matrix eloment between ${ }^{14} \mathrm{C} \otimes s_{\Xi-}$ and ${ }^{13} \mathrm{~B} \otimes s_{\Lambda} p_{\Lambda}$, we require the proton pickup spectroscopic amplitude from ${ }^{14} \mathrm{C}$ (g.s.). In this favorable case, this 

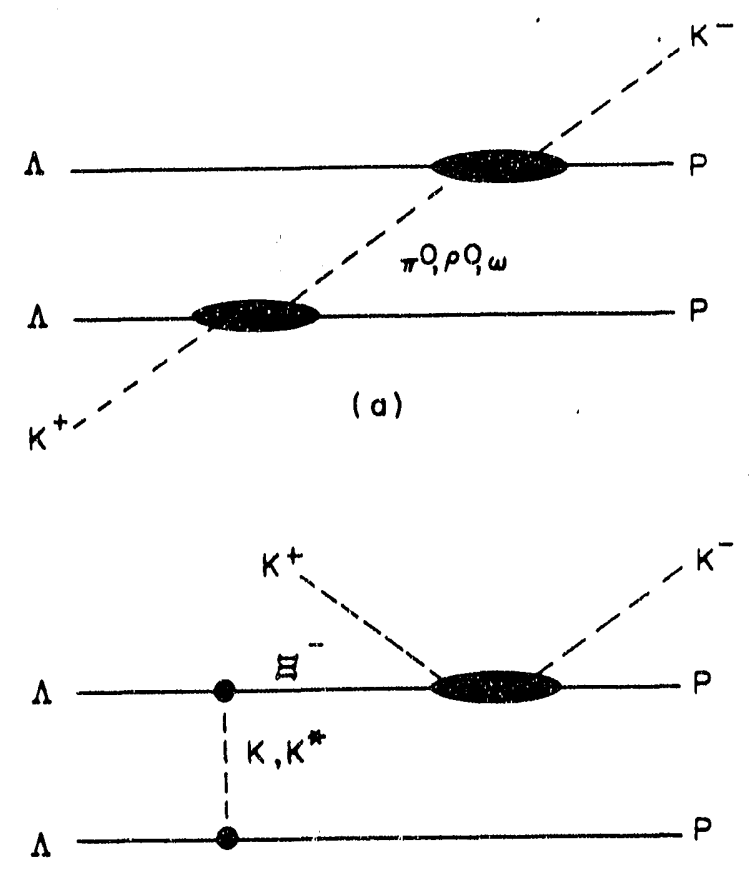

(b)

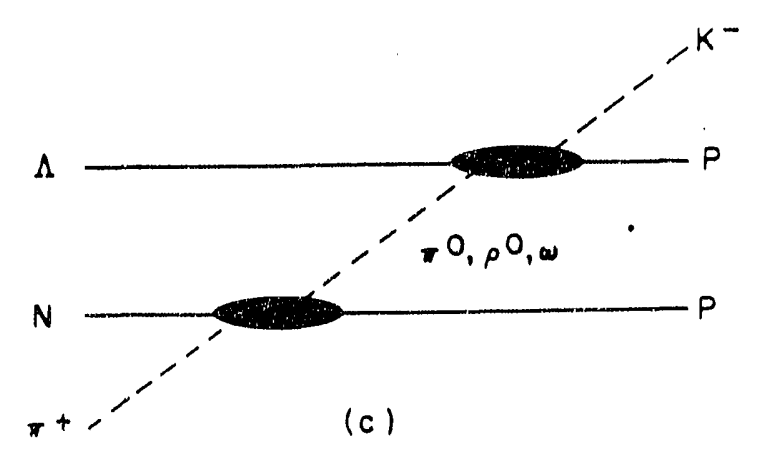

Figure 4: Second order processes for producing $\Lambda \Lambda$ hypernuclei in the $\left(K^{-}, K^{+}\right)$ reaction are shown in (a) and (b). In (c), we show a mechanism for obtaining $\Lambda$ hypernuclei in the $\left(K^{-}, \pi^{+}\right)$process.

is concentrated in the ground state of ${ }^{13} \mathrm{~B}$ : we find $C^{2} S \approx 3.6$, implying that nearly all the $p_{3 / 2}$ protons participate. Including the spectroscopic factor as well as other recoupling coefficients, we find ${ }^{28}$ a mixing amplitude

$$
\alpha \approx 0.67 \frac{\left\langle p_{N} s \Xi|V| s_{\Lambda} p_{\Lambda}\right\rangle}{\Delta E} \approx 0.05\langle V\rangle
$$

where $\langle V\rangle$ is in units of $\mathrm{MeV}$ and the $\Xi N$ and $\Lambda \Lambda$ pairs are both coupled to a spin-isospin singlet state with $L=1$. Denoting by $T_{\Xi}$ and $T_{\Lambda \Lambda}$ the amplitudes for the first and second order $\left(K^{-}, K^{+}\right)$processes, the small admixture $\alpha$ of the $\Xi$ configuration will produce a modified cross section proportional to $\left|T_{\Lambda \Lambda}+\alpha T_{\Xi}\right|^{2}$. Since $\left|T \equiv / T_{\Lambda \Lambda}\right|^{2}$ is of order $10^{2}-10^{3}$, one could obtain enhancement factors of order 4-17 for $\alpha=0.1$, assuming an optimal 


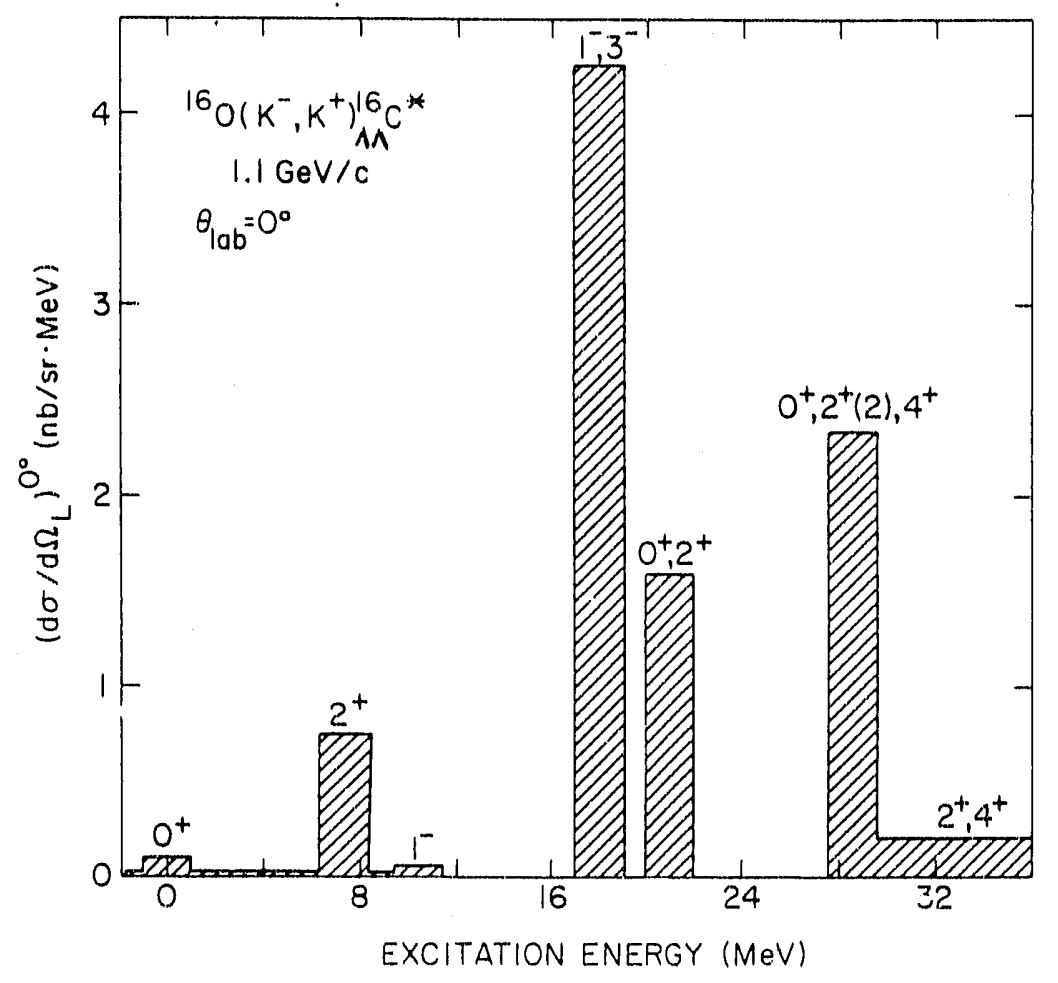

Figure 5: Forward differential cross sections for the excitation of ${ }_{\Lambda \Lambda}^{16} \mathrm{C}$ states in the $\left(K^{-}, K^{+}\right)$reaction on an ${ }^{16} \mathrm{O}$ target ${ }^{76}$. An energy resolution of $2 \mathrm{MeV}$ is assurned.

constructive irterference. In some cases, this could render $\left(K^{-}, K^{+}\right)$experiments feasible at the Brookhaven AGS. Of course, in the long term, the full exploitation of $S=-2$ hypernuclear physics will demand the much higher $\mathrm{K}^{-}$intensities of KAON.

\section{MULTI-STRANGE HYPERNUCLEI AND STRANGELETS}

If we take a heavy nucleus, and start replacing nucleons by $\Lambda$ 's, many units of strangeness can be added before the system becomes unstable ${ }^{30}$. Such systems are natural extensions of $\Lambda$ hypernuclei: they would have binding energies of a few $\mathrm{MeV}$ per particle and weak lifetimes of order $0.2 \mathrm{~ns}$ characteristic of the $\Lambda N \rightarrow N N$ process. There is the exciting possibility ${ }^{31,32}$ that more strongly bound multi-strange conglomerates, called "strangelets", may exist. Unlike a loosely bound system of $\Lambda$ 's, a strange quark in a strangelet roams over the entire volume of the confining bag, and is hence not correlated with a $(u d)_{I=0}$ diquark to form a $\Lambda$. Berger and Jaffe ${ }^{33}$ have provided a strangelet binding energy formula of the form

$$
\begin{aligned}
B(A, Y, Z) / A & =a_{V}-a_{S} / A^{1 / 3}-\left(\frac{a_{C}}{A^{4 / 3}}+\frac{\delta_{Z}}{2 A^{2}}\right)\left(Z-Z_{\min }\right)^{2} \\
& -\frac{1}{2} \frac{\delta_{Y}}{A^{2}}\left(Y-Y_{\min }\right)^{2}
\end{aligned}
$$




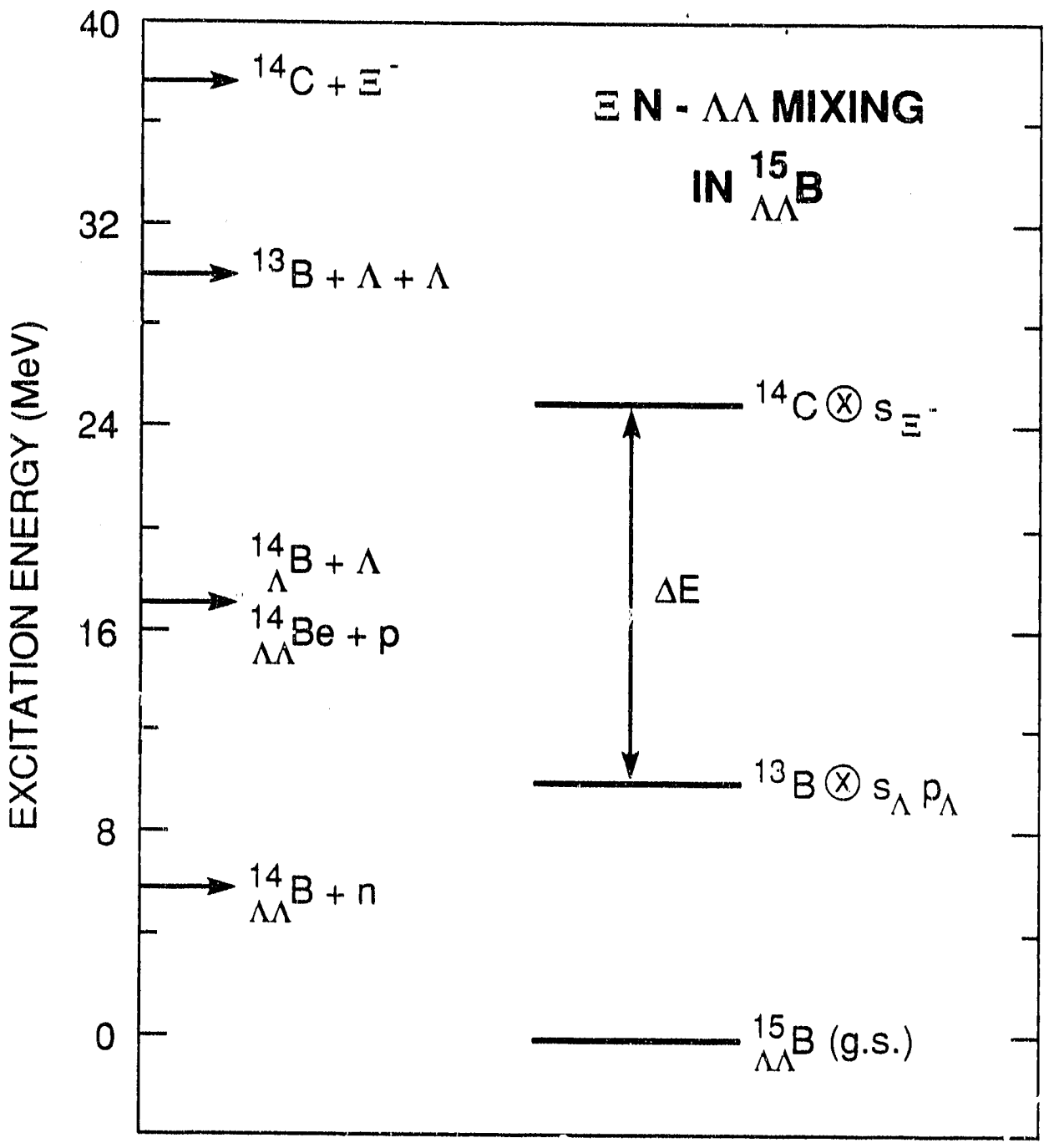

Figure 6: Partial excitation energy spectrum, with particle emission thresholds, for the ${ }_{\Lambda \Lambda}^{15} \mathrm{~B}$ double hypernucleus. $\Delta E \approx 15 \mathrm{MeV}$ indicates the energy separation of two levels mixed by the $\Xi N \rightarrow \Lambda \Lambda$ transition potential.

In addition to volume $\left(a_{V}\right)$, surface $\left(a_{S}\right)$ and Coulomb $\left(a_{C}\right)$ terms whicis also occur in the usual nuclear mass formula, Eq. (13) contains a term dependent on the hypercharge $Y$. For ordinary nuclei, we have $a_{V}=15.5 \mathrm{MeV}$, the binding energy per particle of infinite nuclear matter. In Eq. (13), the various constants depend on two parameters of the MIT Bag model, namely the strange quark current mass $m_{s}$ and the bag pressure $B$.

The only practical way of producing multi-strange hypernuclei or strangelets in the laboratory is by means of relativistic heavy ion collisions. In an encounter between heavy ions, each of the independent nucleon-nucleon collisions is capable of producing an $s \bar{s}$ quark pair. It is already known from AGS experiments at $15 \mathrm{GeV} / \mathrm{A}$ that substantial numbers of strange particles are produced in heavy ion central collisions ${ }^{34}$. Searches of modest sensitivity for long-lived stranglets have already been performed ${ }^{35}$, with negative results. However, to obtain a meaningful test for the existence of strangelets, very high sensitivity measurements 
are required. This is the goal of experiment E864 at Brookhaven ${ }^{36}$, which can attain sensitivities in the range $10^{-10}$ to $10^{-11}$ per collision for the detection of a variety of long-lived $(\tau>5-10 \mathrm{~ns})$ objects of either positive or negative charge.

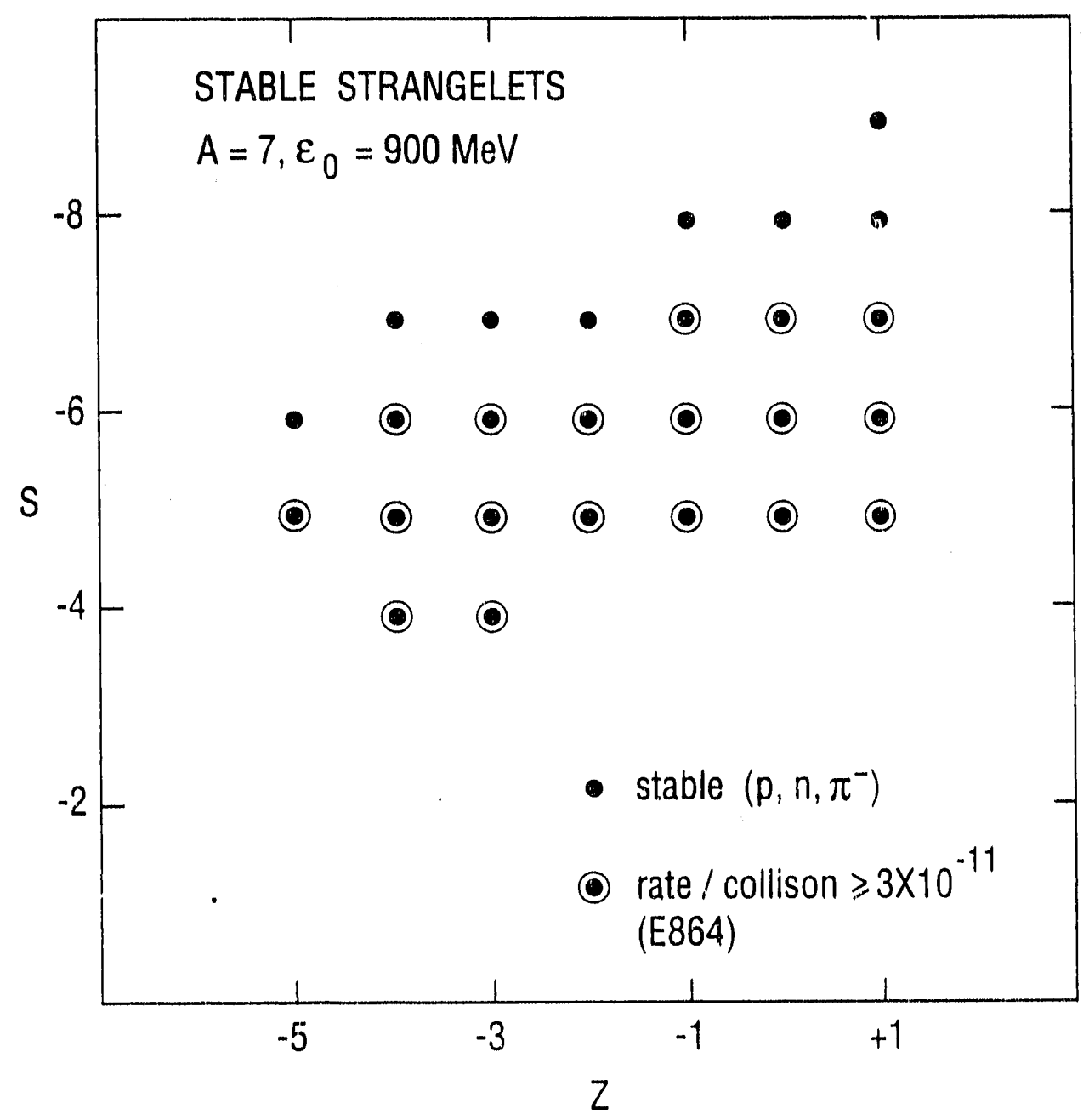

Figure 7: Stability region of strangelets in the plane of strangeness $S$ and charge $Z$, for fixed baryon number $A=7$. A strange quark mass $m_{s}=150$ $\mathrm{MeV}$ and a volume energy $\epsilon_{0}=m_{N}-a_{V}=900 \mathrm{MeV}$ was used in tise BergerJaffe mass formula. The black dots indicate the species which are stable with respect to both strong and weak neutron, proton and $\pi^{-}$emission. The circled dots indicate those strangelets which could be produced at rates larger than the E864 sensitivity of $3 \times 10^{-11}$ in $\mathrm{Au}+\mathrm{Au}$ collisions at Brookhaven AGS energies $(11 \mathrm{GeV} / \mathrm{A})$, according to the coalescence estimates of Ref. 38 .

There exist no quantitative estimates of strangelet production rates in heavy ion collisions, but there are some speculations based on the distillation of strangeness in the hadronization of quark-gluon plasma ${ }^{37}$ or more conventional multi-baryon coalescence ${ }^{38}$. For a particular choice of parameters $\left(\iota_{V}=40 \mathrm{MeV}\right)$ in $\mathrm{El}_{1}$. (13), the region of strangeness and charge for which $A=7$ strangelets are stable against strong and weak emission of $n, p$ 
or $\pi^{-}$is shown in Fig. 7. Based on the crude coalescence estimates ${ }^{38}$ of rates, the species which could be detected in experiment $\$ 864$ are indicated as circled dots. It has been emphasized by Jaffe ${ }^{32,33}$ that Eq. (13) may not be valid for small $A$, since it represents an expansion in powers of $A^{-1 / 3}$. In the coalescence picture, the maximum strangeness that one can effectively attain is considerably more modest $(|S| \leq 7$ in Fig. 7$)$ than that predicted if quark-gluon-plasma is formed ${ }^{37}$. In the future, a strangelet search the Relativistic Heavy Ion Collider RHIC should be mounted. If strangelets are indeed sule, they may represent the best signal for the transient formation of the plasma!

\section{CONCLUSIONS}

Strange particle nuclear physics is an exciting and vibrant field of research. On the experimental front, one can anticipate qualitacive advances in our knowledge of hypernuclear spectroscopy with the advent of CEBAF, and perhaps later PILAC and KAON. These facilities will offer an entry into the domain of high resolution spectroscopy: only then will we gain access to the details of the spin and flavor dependence of baryon-baryon interactions, i.e. the roble of the strangeness degree of freedom in strong interaction dynamics. Construction

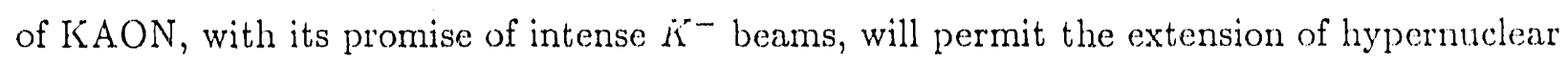
structure studies from the familiar $S=-1$ sector to the essentially uncharted terrain of $S=-2$ systems. For the study of multi-strange objects with $S \leq-3$, relativistic heavy ion collisions are the method of choice, since a copious bath of strange particles can occasionally result from a central collision. Such collisions represent our best chance for producing strange quark matter in the laboratory, if indeed relatively small droplets of this exotic substance are stable.

\section{REFERENCES}

1. R. Engelmann et al., Phys. Rev. 21 (1966) 587;

B. Sechi-Zorn et al., Phys. Rev. 175 (1968) 1735;

J.A. Kadyk et al., Nucl. Phys. B27 (1971) 13.

2. C.B. Dover and H. Feshbach, Ann. Phys. 198 (1990) 321.

3. M.M. Nagels, T.A. Rijken and J.J. deSwart, Phys. Rev. D12 (1975) 744; D15 (1977) 2547; D20 (1979) 1633.

4. P.M.M. Maessen, T.A. Rijken and J.J. deSwart, Phys. Rev. C40 (1989) 2226.

5. U. Straub et al., Nucl. Phys. A483 (1988) 686;

Y. Koike, K. Shimizu and K. Yazaki, Nucl. Phys. A513 (1990) 653.

6. B. Holzenkamp, K. Holinde and J. Speth, Nucl. Phys. A500 (1989) 485.

7. W. Brückner et al., Phys. Lett. 79B (1978) 157.

8. R.E. Chrien et al., Phys. Rev. C41 (1990) 1082.

9. C.B. Dover and H. Feshbach, Ann. Phys. (in press).

10. A.R. Bodmer and Q.N. Usmani, Nucl. Phys. A477 (1988) 621.

11. D.J. Millener, A. Gal, C.B. Dover and R.H. Dalitz, Phys. Rev. C31 (1985) 499.

12. V. Fetisov et al., Z. Phys. A339 (1991) 399. 
13. H.A. Thiessen, in Proc of the LAMPF Workshop on $(\pi, K)$ Physics, AIP Conf. Proc.

No. 224, Part. and Fields Series 43, Eds. B.F. Gibson et al., New York (1991), p. 49.

14. J. Cohen, Intern. J. of Mod. Phys. A4 (19.39) 1.

15. M. Bedjidian et al., Phys. Lett. B83 (1980) 252.

16. C.B. Dover and D.J. Millener, in Modern Topics in Electron Scattering, Eds. B. Frois and I. Sick, World Scientific, Singapore (1991), pp. 608-644.

17. P.H. Pile et al., Phys. Rev. Lett. 66 (1991) 2585.

18. D.J. Millener, C.B. Dover and A. Gal, Phys. Rev. C38 (1988) 2700.

19. Y. Yamamoto, H. Bandō and J. Z̆ofka, Prog. Theor. Phys. 80 (1988) 757.

20. C.B. Dover, D.J. Millener, and A. Gal. Phys. Rep. 184 (1989) No. 1, pp. 1-97.

21. R.L. Jaffe, Phys. Rev. Lett. 38 (1977) 195.

22. M. Danysz et al., Nucl. Phys. 49 (1963) 121;

J. Prowse, Phys. Rev. Lett. 17 (1966) 782.

23. S. Aoki et al., Prog. Theor. Phys. 85 (1991) 1287.

24. C.B. Dover, D.J. Millener, A. Gal and D.H. Davis, Phys. Rev. C44 (1991) 1905.

25. D. Zhu et al., Phys. Rev. Lett. 67 (1991) 2268.

26. A.J. Baltz, C.B. Dover and D.J. Millener, Phys. Lett. 123B (1983) 9.

27. C.B. Dover and A. Gal, Ann. Phys. 146 (1983) 309.

28. C.B. Dover, A. Gal and D.J. Millener, in preparation.

29. Y. Akaishi and K.S. Myint, in these Proceedings.

30. M. Rufa et al., Phys. Rev. C42 (1990) 2469.

31. E. Witten, Phys. Rev. D30 (1984) 272.

32. E. Farhi and R.L. Jaffe, Phys. Rev. D30 (1984) 2379; D32 (1985) 2452.

33. M.S. Berger and R.L. Jaffe, Phys. Rev. C35 (1987) 213; to connect with our notation: $a_{V}=r \cdot N-\epsilon_{0}, a_{S}=4 \pi \sigma r_{0}^{2}, a_{C}=3 \alpha / 5 r_{0}$.

34. O. Hansen, Comments Nucl. Part. Phys. 20 (1991) 1.

35. J. Barrette et al., Phys. Lett. 252B (1990) 550.

36. Brookhaven AGS experiment E864: Production of Rare Composite Objects in Relativistic Heavy Ion Collisions, J. Sandweiss and R. Majka, Spokespersons.

37. C. Greiner and H. Stöcker, Phys. Rev. D44 (1991) 3517.

38. C.B. Dover, Proc. Intersections between Particle and Nuclear Physics, Tucson, Arizona, May 1991, AIP Conf. Proc., Particles and Fields Series, in press.

\section{DISCLAIMER}

This report was prepared as an account of work sponsored bv an agency of the United States Government. Neither the United States Government nor any agency thereof, nor any of their employees, makes any warranty, express or implied, or assumes any legal liability or responsibility for the accuracy, completeness, or usefulness of any information, apparatus, product, or process disclosed, or represents that its use would not infringe privately owned rights. Reference herein to any specific commercial product, process, or service by trade name, trademark, manufacturer, or otherwise does not necessarily constitute or imply its endorsement, recommendation, or favoring by the United States Governmenl or any agency thereof. The views and opinions of authors expressed herein do not necessarily state or reflect those of the United States Government or any agency thereof. 

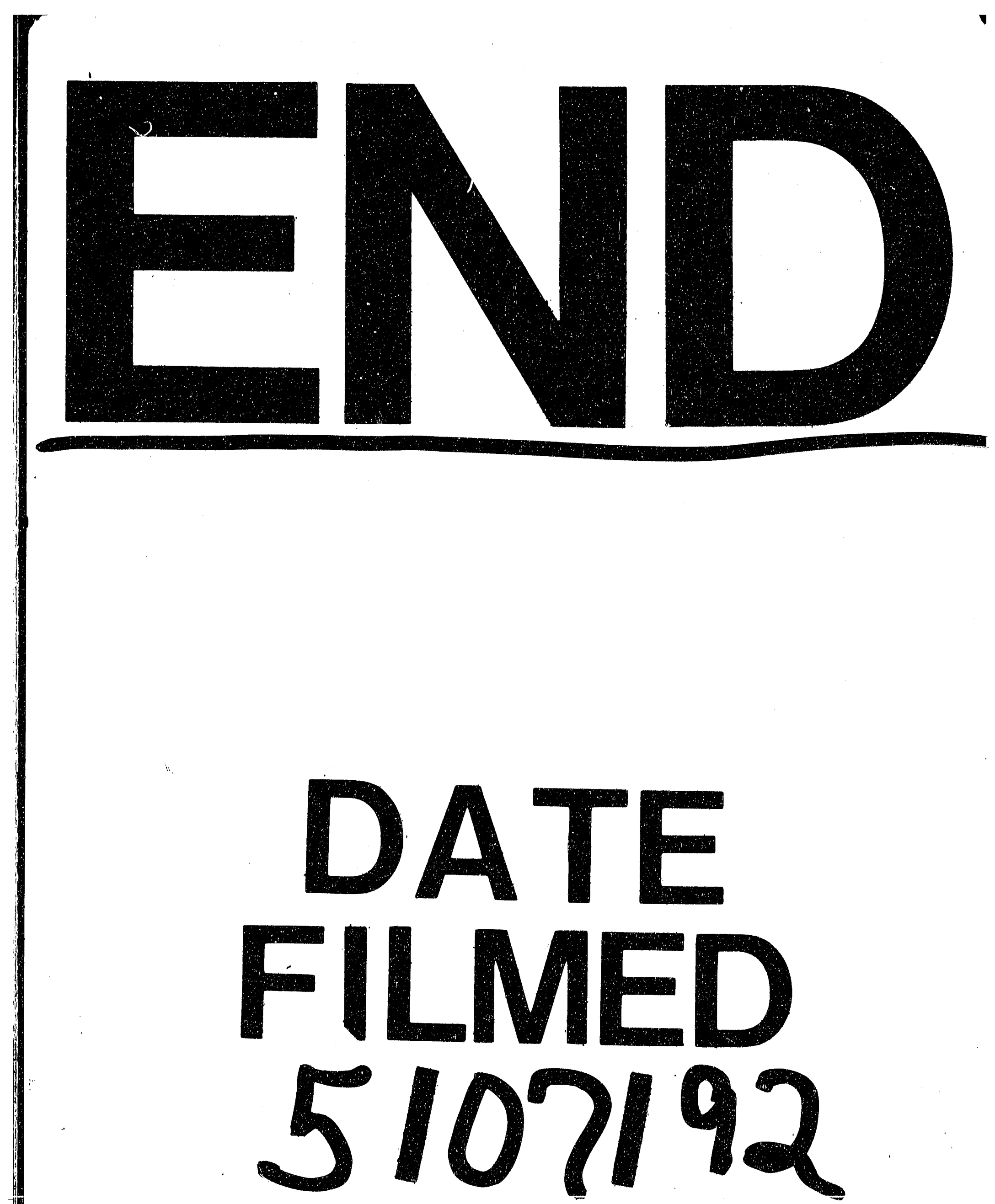
